\title{
The effect of increasing the contact surface on tendon healing
}

\author{
Fatma Bilgen ${ }^{1}$, Yakup Duman ${ }^{1}$, Ömer Bulut ${ }^{2}$, Mehmet Bekerecioğlu ${ }^{1}$ \\ ${ }^{1}$ Department of Plastic, Reconstructive, and Aesthetic Surgery, Kahramanmaraş Sutcu Imam University School of Medicine, Kahramanmaraş; \\ ${ }^{2}$ Department of Plastic, Reconstructive, and Aesthetic Surgery, Gaziantep 25 December Hospital, Gaziantep, Turkey
}

Background The most common complication after tendon repair is the development of adhesion, with subsequent rupture.

Methods In this study, we present a new method in which the tendon healing contact surface is increased to reduce these complications. The tendons of chickens in groups 1, 3, and 5 were transversely cut and repaired with in the traditional fashion with double-modified Kessler method and 5/0 polypropylene. In the other groups, $3 \mathrm{~mm}$ of the tendon was removed from the proximal half of the upper end and from the distal half of the lower end of the tendon, and they were repaired with the modified Kessler method. The tendons of the chickens in groups 1 and 2 were evaluated immediatelly after surgery. Groups 3 and 4 were evaluated at 4 weeks after surgery. Groups 5 and 6 were evaluated at 6 weeks.

Results Increases in transient inflammation and connective tissue formation were observed more clearly in the group treated with the new method in histopathological investigations at weeks 4 and 6 . The stretching test showed statistically significant differences between groups 3 and $4(\mathrm{P}<0.05)$ and groups 5 and $6(\mathrm{P}<0.05)$.

Conclusions When repairing tendons with the new method, the healing surface increases and the direction of collagen fibers at the surface changes. Because of these effects, the strength of the tendon healing line increases; we therefore expect that this technique will enable patients to safely engage in early active exercise after the operation, with less risk of tendon rupture.

Keywords Tendons / Wounds and injuries / Fibrosis / Movement
Correspondence: Fatma Bilgen Department of Plastic, Reconstructive, and Aesthetic Surgery, Kahramanmaraş Sutcu Imam University School of Medicine, Avsar neighborhood, West, Number: 251/A 46040 Kahramanmaraş, Turkey

Tel: $+90-5057280165$

Fax: $+90-3443003409$

E-mail: fatmabilgen81@gmail.com

Received: 29 Jul 2017 • Revised: 16 Apr 2018 • Accepted: 30 Apr 2018

pISSN: 2234-6163 • elSSN: 2234-6171・ https://doi.org/10.5999/aps.2017.01284• Arch Plast Surg 2018;45:357-362

This article was adapted from the Ömer Bulut MD thesis from Gaziantep Üniversity (Turkey) in 2006.

\section{INTRODUCTION}

Tendon injuries are an important aspect of the practice of hand surgery. The use of a suture technique that is strong, non-spaced and does not cause any adhesions on the tendon surface and pulleys is a major factor that helps patients regain their strength and restore their hand movements following tendon repair [1-3]. The main principle in end-to-end tendon repair is to provide a smooth healing surface without causing limitations of movement in the tendon. Therefore, many tendon repair methods have been developed and described. The most common complication of tendon repair is tendon adhesion, with subsequent rupture. Early active exercise is initiated to prevent adhesion. In the postoperative period, when the tendon repair line is the weakest, tendon rupture may occur during active exercise [4-6]. Repair techniques and suture materials can also cause ruptures. 
In the present study, we developed a new tendon repair method, enhancing the tendon healing surface to prevent ruptures that may occur during active exercise, with the goal of improving healing in the postoperative period.

In the present experimental study conducted in a chicken model, we aimed to increase the strength of the tendon repair line by changing the direction of tendon healing and forming new cruciate fibers in addition to linear collagen fibers, as well as by increasing the number of collagen fibers and fibroblasts by increasing the tendon healing surface. Through both effects, we aimed to decrease the likelihood of tendon rupture and adhesions that may occur in the postoperative period when patients start early active exercise.

\section{METHODS}

This study was conducted with the approval of the Gaziantep Medical Faculty Local Ethics Board (No. 2006/1888).

\section{Preparation}

Chicken flexor tendons are preferred as an animal model because of their similarity to the human hand in terms of anatomi$\mathrm{cal}$ and functional properties and their easy availability. Leghorn-type white chickens were used in this study (14-16 months old, weighing 1,750-2,000 g). The chickens were randomly divided into six groups and there were eight chickens in each group. The subjects were observed for 1 week in their separate cages before study. No food was given the night before the study. After processing, a splint was applied, and they were returned to their natural environment. During the study, they were fed in compliance with the H4-line white W-77 protocol.

The right legs of the chickens in all groups underwent operations, and numbers were attached to their left feet. After the surgery, the chickens were put into cages in four groups.

\section{Surgical technique}

$\mathrm{HCl}$ (12.5 mg/kg, Ketalar 5\%, Eczacibasi, Istanbul, Turkey) was administered intramuscularly to the pectoral muscles of chickens, which had their group number attached to their left feet.

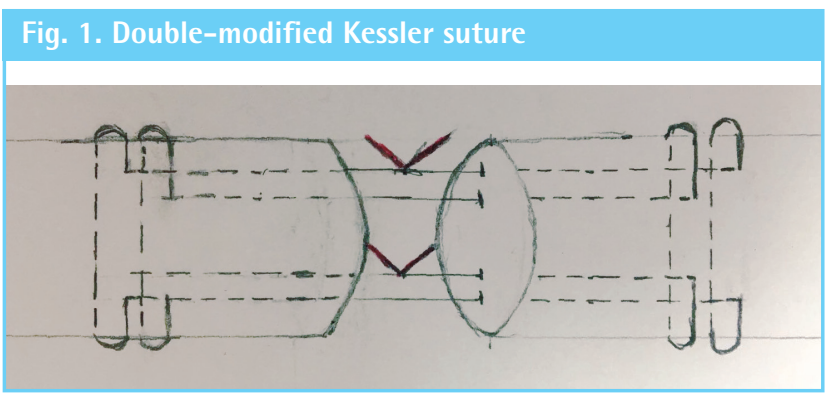

Additionally, $250 \mathrm{mg}$ of cefazolin sodium (Cefozin; Bilim Pharmaceuticals, Istanbul, Turkey) was injected intramuscularly in each subject, and preoperative prophylaxis was then performed.

The right legs of all chickens were washed with Isosol (Scrub), and then sterilized with Biocadin. The flexor digitorum profundus tendon was accessed through a Bruner zigzag incision extending $2 \mathrm{~cm}$ from the region appropriate for the proximal phalanx towards the distal aspect, removing the skin flaps. The tendons of the subjects in groups 1,3 , and 5 were transversely cut and repaired using the double-modified Kessler method with $5 / 0$ round polypropylene (Prolene) sutures, and these groups were used as controls. The novel tendon repair method analyzed in this study was performed in the subjects in groups 2,4 , and 6 . In this method, tendons were repaired in a step-like manner by the double-modified Kessler method after the removal of $3 \mathrm{~mm}$ of the tendon region from the upper half of the proximal end of the tendon and $3 \mathrm{~mm}$ from the lower half of the distal end (Figs. 1 and 2).

\section{Measurements}

Stretching tests of the chickens in group 1 and group 2 were performed by dislodging the legs under the knee following the repair. Immobilization was provided by placing plaster on the right foot of the chickens in standing position in accordance with their walking physiology. Stretching tests of the tendons of the chickens in groups 3 and 4 were performed at 4 weeks after surgery. The stretching tests were performed at 6 weeks in groups 5 and 6 . At 4 and 6 weeks, the tendons of chickens randomly taken from the groups were sent to a pathology laboratory for histopathological examinations.

All tendon repairs were performed with the double-modified

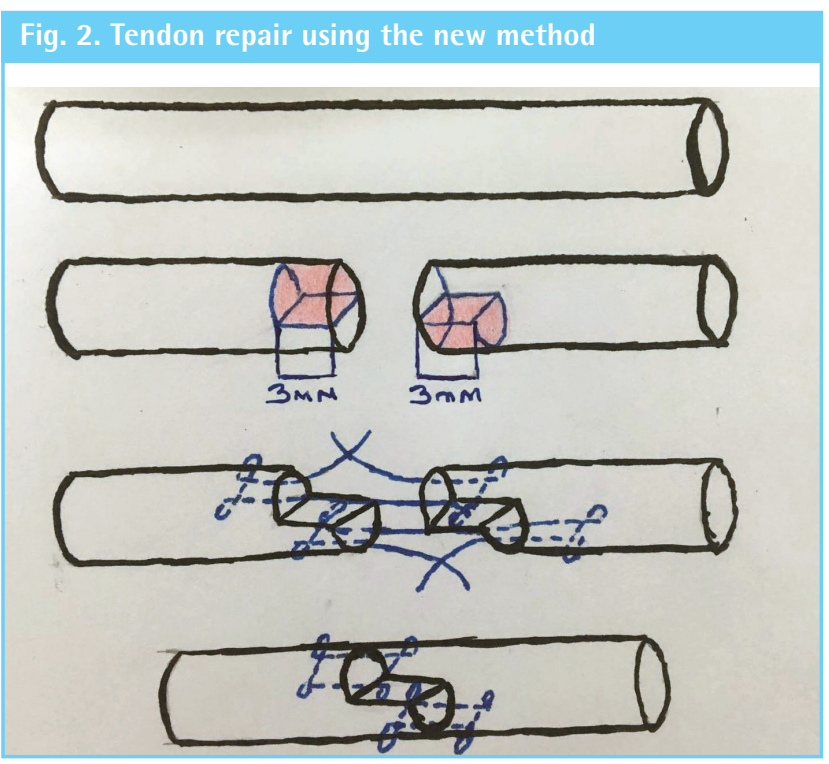


Kessler method and round 5/0 polypropylene sutures. After surgical operations, the subjects were sacrificed through an intramuscular injection of pentobarbital $(100 \mathrm{mg} / \mathrm{kg})$.

Data were obtained through a gross examination, histopathological examination (with hematoxylin and eosin staining) and biomechanical examinations (Testometric Micro 500 device, Rochdale, England), and a statistical analysis was then conducted.

In the gross examination, the tendons were examined through the previous incision scar. Three centimeters of the tendon had been removed from the proximal and distal ends. Whether there was a gap in the suture line was observed.

In biomechanical examinations, the amount of force needed for the tendon to start to rupture, for a 2-mm gap to occur, and for the tendon to completely rupture was determined and recorded by fixing both ends of the tendons on the Testometric device.

For the histopathological examinations, paraffin block sections were taken from tendons routinely stored in $10 \%$ formaldehyde after the operations. Hematoxylin and eosin staining was used to evaluate the specimens for the presence of fibrosis, inflammation, and intravenous inflammatory cells.

\section{Statistical analysis}

Statistical analysis was performed using the Minitab program (Sigma, Turkey) on a personal computer. Non-parametric variables were analyzed using the Mann-Whitney $\mathrm{U}$ test to identify significant intragroup and intergroup differences. Parametric tests (mean) were applied to the groups as appropriate. A P-value of 0.05 was considered to indicate statistical significance.

\section{RESULTS}

Results of the biomechanical study: For each group, the magni-

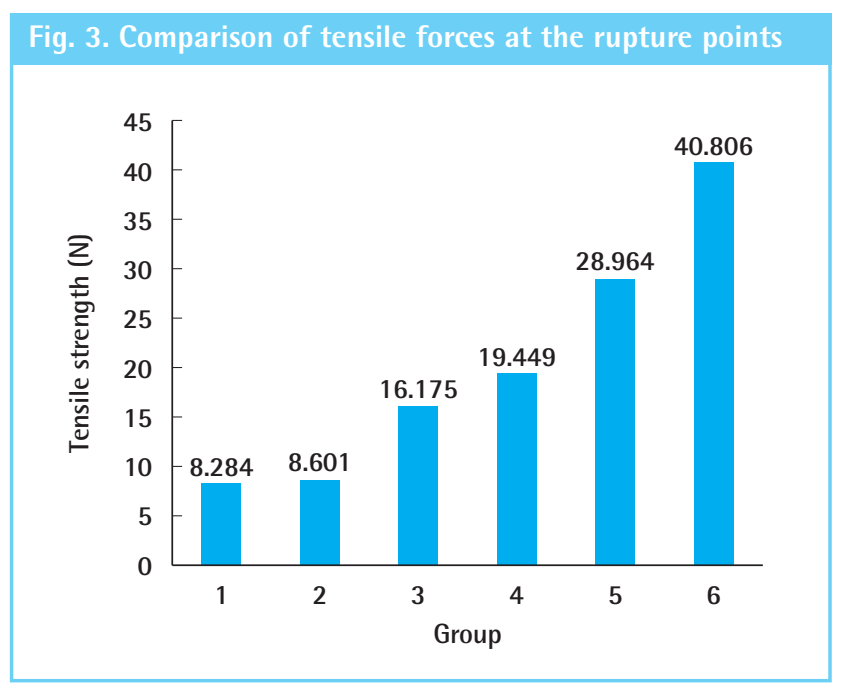

tude of the force (in Newtons) needed for the tendon to start to rupture, for a 2-mm gap to occur, and for the tendon to completely rupture was recorded. There was no significant difference in the stretching test results of the chickens between group 1 and group $2(\mathrm{P}>0.05)$. However, statistically significant differences in the stretching test results were found between groups 3 and $4(\mathrm{P}<0.05)$ and groups 5 and $6(\mathrm{P}<0.05)($ Table 1$)$.

Group 1 had the lowest tensile strength, and group 6 had the highest (Fig. 3). The new method was found to lead to stronger tendons than the traditional double-modified Kessler method (Fig. 4).

Histopathological results: fibrosis, inflammation, and intravenous inflammatory cells were evaluated along the tendon repair line (Table 2).

There was no significant difference in the proportion of specimens showing inflammation between groups 3 and 4 at 4 weeks; however, the number of intravenous inflammatory cells was higher in group 4. At 6 weeks, there were no intravenous inflammatory cells in groups 5 and 6 . However, group 6 had more inflammation than group 5, while group 6 had more fibrosis than group 5 (Figs. 5 and 6).

These results show that an increase in transient inflammation

\begin{tabular}{|c|c|c|c|}
\hline Group & Week 0 & Week 4 & Week 6 \\
\hline 1 & $8.284 \pm 0.389$ & - & - \\
\hline 2 & $8.601 \pm 0.411$ & - & - \\
\hline 3 & - & $16.175 \pm 0.411$ & - \\
\hline 4 & - & $19.449 \pm 0.443$ & - \\
\hline 5 & - & - & $28.964 \pm 0.493$ \\
\hline 6 & - & - & $40.806 \pm 0.775$ \\
\hline
\end{tabular}

\section{Fig. 4. Time-dependent tensile strengths}

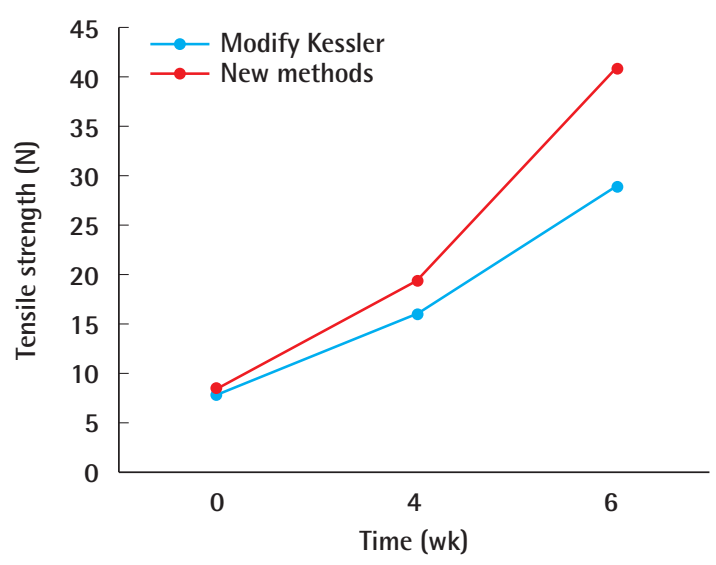


and connective tissue formation was an indication of an increase in the healing surface. Increased fibrosis tissue was found to contribute to healing and increased firmness.

\section{DISCUSSION}

Tendon injuries are commonly encountered in daily life. Studies in this field have primarily focused on tendon repair techniques and their effects on tendon healing, as well as on early mobilization of the tendon repair area (i.e., the healing of non-tendon factors) [1-4].

For ideal tendon repair, restoring the tendon sheath, not leaving a space on the suture line, using an appropriate suture technique, and providing appropriate immobilization and early controlled movement are required $[4,5]$.

The most important problems encountered after tendon repair are adhesion and rupture $[3,4]$. In the formation of anti-slip adhesions after flexor tendon injury and repair, the rate of collagen synthesis plays an important role during healing, and consequently, relatively more or less adhesion will occur following flexor tendon injuries and repair. Several studies have investigated whether various biochemical agents and interpositional materials could prevent adhesions during tendon healing [5-7].

Table 2. Evaluation of histopathological parameters in the
various groups
\begin{tabular}{|lcrcc|}
\hline Group & Fibrosis & Inflammation & $\begin{array}{c}\text { Intravenous } \\
\text { inflammatory cells }\end{array}$ \\
\hline 3 & + & ++ & + \\
4 & + & ++ & ++ \\
5 & + & ++ & - \\
6 & +++ & +++ & - \\
\hline
\end{tabular}

\section{Fig. 5. Histopatological image of group 5}

Fibrosis and inflammation found in tissue (hematoxylin and eosin, $\times 100)$.

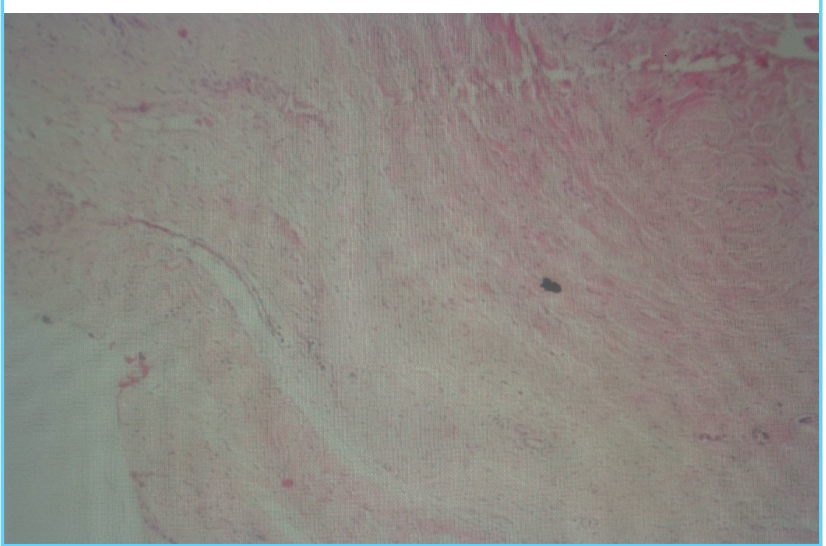

The most important causes of adhesion after tendon repair are tendon sheath injuries, improper suturing, and immobilization. If the repaired flexor tendons are not under stress, the healing process may extend for up to 8 weeks, and the tensile strength will be minimized in the tendons that have been repaired during the healing period. Stressed tendons heal faster than unstressed tendons and gain tensile strength faster, and these tendons have less adhesion and more excursion $[7,8]$.

Exercise is the most important factor preventing adhesion after tendon repair. In general, passive exercises have been used to prevent adhesion after tendon repair $[9,10]$. Early movement is very important after tendon repair.

In a study performed on dogs, Gelberman et al. [11] showed that tendon healing was accelerated through early mobilization, with fewer adhesions. In the postoperative period, having patients engage in active exercises, rather than passive exercises, is recommended to prevent adhesion. Since passive exercises are insufficient for tendon repair, early active exercises are initiated; additionally, tendon slipping is increased, adhesion is prevented by postoperative active exercise [11-13].

Suture techniques are another important factor in tendon repair. There are many central suture methods in tendon repair. Tendon sutures such as the Kessler suture and its modifications are predominant in terms of preventing gap formation and rupture in the recovery period $[14,15]$. The rounded suture recommended by Tsuge et al. [16] is particularly useful for suturing a ruptured limb, since it can be performed rapidly and is as resistant to rupture as the Kessler method. Since the modified Kessler method, which leaves the knot inside the tendon, is the most common tendon suture, we used this suture method in our technique. Tendon repair strength is mainly determined by the central suture. The epitendinous suture contributes to the

\section{Fig. 6. Histopatological image of group 6}

Fibrosis and inflammation found in tissue (hematoxylin and eosin, $\times 100)$.

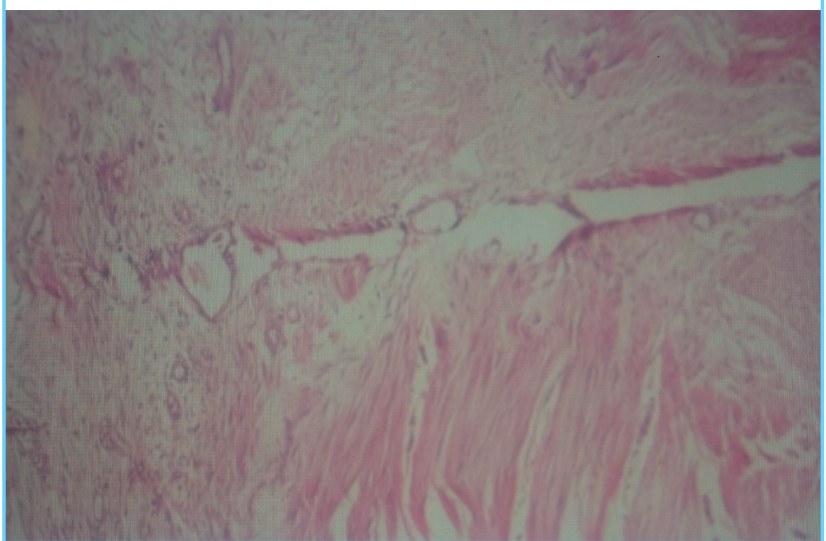


strength of the tendon [17].

A study by Cao and Tang [18] in 2005 showed that multiband repair minimized tendon ruptures, and active exercise could be performed without tendon ruptures or gap formation due to tendon regression and suture relaxation $[18,19]$.

Following tendon repair, a softening occurs in the repair line in the early period, and the suture easily peels from the tendon. The resistance of the suture line against the external forces is decreased in the first 5 days, and declines to $20 \%$ of its value on the first day. However, it eventually recovers much of its former strength. Partial formation of the tendon at the tendon end is more frequent than tendon rupture. This gap, which is not apparent from the outside, causes an increase in adhesions and leads to poor results [17-19].

One of the most important factors contributing to the durability of repaired flexor tendons is the number and diameter of the sutures at the repair area. A higher number of sutures passing through the repair area increases the durability of the repair $[15,20]$.

Although the Kessler technique is currently the most popular method, many alternative suture techniques exist [20]. In a study of fresh pig cadavers in 2003, Wang et al. [21] biomechanically compared the forces required to form a 2-mm gap and to cause rupture between the 6-strang Tang method and the modified Tang method (modified by a horizontal $\mathrm{M}$ suture in addition to the 6-strang suture), and the tensile strength of the tendons repaired with the modified technique was found to be higher.

In a study by Cao and Tang [18], a biomechanical comparison was conducted of the forces required for gap formation, 2-mm gap formation, and complete rupture between the 4-band modified Tang suture and the double Kessler suture, and the tensile strength of the modified Tang method was found to be higher [17-22].

The most important step after tendon repair is to start postoperative active exercises. At the end of the second week after tendon repair, the strength of the suture is reduced and a gap is formed due to the regression of tendon edema. During this period, collagen bridges begin to form between the healing surfaces of the tendon. The most important factor at this point is to increase the tendon healing capacity by maintaining this gap. This capacity is directly related to the amount of fibroblast and collagen fibers in the environment [20-22].

Active exercise increases fibroblast proliferation and collagen synthesis [21-23]. Additionally, a large healing area results in more collagen production. In our method, the tendon ends were sutured in the form of a ladder step, and thus the collagen fibers were arranged in different directions on the tendon healing sur- face, resulting in the formation of cruciate fibers. These cruciate fibers increase the strength of the tendon by forming tight bonds during tendon healing $[14,23]$. Furthermore, in the new method, the tendon healing surface was increased by the extraction component of the procedure. An extraction of three times the radius of the tendon doubles the healing surface, resulting in at least twice as much collagen as normal. In the method we have described, histopathologic fibrosis and inflammation were found to increase even more in the tendons. For this reason, the active exerciser can be started earlier with active exercise, fibroblasts produce collagen, which is why a much larger healing surface was created in our technique than in other methods.

In Z-plasty and W-plasty methods, less power is applied to the healing surface since the direction of the stress is changed, and the risk of tendon rupture is thereby decreased. In our technique, the stress on the tendon healing line also decreases as the area of each healing surface decreases.

In the new technique, tendon length is shortened by up to 3 $\mathrm{mm}$. However, this is not a disadvantage in primary tendon repair or tendon grafting. In late tendon repair, this method may be challenging due to tendon shortening. In such cases, if the tendon is repaired, regardless of the method, the patient should be given passive exercises due to the risk of tendon rupture during active exercise [24].

In case of fibrillated tendon ends, it may be necessary to remove some of the ends of the tendon to make a good suture. In such cases, it is not problematic to remove up to $1 \mathrm{~cm}$. More shortening can lead to contractures in the fingers [24].

Hashimoto et al. [25] modified proximal tendon-to-tendon repair with a step-cut (SC) technique that yielded similar repair strength $[23,24]$. Becker et al. reported an innovative bevel-cut technique, similar to the SC technique, to eliminate gap formation following primary tendon repair. The ultimate failure load of the technique of Becker et al. was higher than that of the Kessler and Bunnell repairs [24].

In our study, we found that increasing the tendon healing surface instead of the central suture number was effective in increasing the healing power in the postoperative period and made a greater contribution to tendon stability. Since active movement increases tendon healing in the early postoperative period, our technique may be more appropriate in primary tendon repair than other methods for preventing adhesion.

\section{NOTES}

\section{Conflict of interest}

No potential conflict of interest relevant to this article was reported. 


\section{Ethical approval}

The study was approved by the Gaziantep Medical Faculty Local Ethics Board (No. 2006/1888) and performed in accordance with the principles of the Declaration of Helsinki.

\section{REFERENCES}

1. Peltz TS, Haddad R, Scougall PJ, et al. Influence of locking stitch size in a four-strand cross-locked cruciate flexor tendon repair. J Hand Surg Am 2011;36:450-5.

2. Sandvall BK, Kuhlman-Wood K, Recor C, et al. Flexor tendon repair, rehabilitation, and reconstruction. Plast Reconstr Surg 2013;132:1493-503.

3. Moriya K, Yoshizu T, Maki Y, et al. Clinical outcomes of early active mobilization following flexor tendon repair using the six-strand technique: short- and long-term evaluations. J Hand Surg Eur Vol 2015;40:250-8.

4. Frueh FS, Kunz VS, Gravestock IJ, et al. Primary flexor tendon repair in zones 1 and 2: early passive mobilization versus controlled active motion. J Hand Surg Am 2014;39: 1344-50.

5. Farzad M, Layeghi F, Asgari A, et al. A prospective randomized controlled trial of controlled passive mobilization vs. place and active hold exercises after zone 2 flexor tendon repair. Hand Surg 2014;19:53-9.

6. Strickland JW. Development of flexor tendon surgery: twenty-five years of progress. J Hand Surg Am 2000;25:214-35.

7. Tang JB, Wang B, Chen F, et al. Biomechanical evaluation of flexor tendon repair techniques. Clin Orthop Relat Res 2001;(386):252-9.

8. Akasaka T, Nishida J, Araki S, et al. Hyaluronic acid diminishes the resistance to excursion after flexor tendon repair: an in vitro biomechanical study. J Biomech 2005;38:503-7.

9. Karakurum G, Buyukbebeci O, Kalender M, et al. Seprafilm interposition for preventing adhesion formation after tenolysis. An experimental study on the chicken flexor tendons. J Surg Res 2003; 113:195-200.

10. Strickland JW, Glogovac SV. Digital function following flexor tendon repair in Zone II: a comparison of immobilization and controlled passive motion techniques. J Hand Surg Am 1980;5:537-43.

11. Gelberman RH, Vande Berg JS, Lundborg GN, et al. Flexor tendon healing and restoration of the gliding surface: an ultrastructural study in dogs. J Bone Joint Surg Am 1983;65: 70-80.
12. Small JO, Brennen MD, Colville J. Early active mobilisation following flexor tendon repair in zone 2. J Hand Surg $\mathrm{Br}$ 1989;14:383-91.

13. Tang JB, Gu YT, Rice K, et al. Evaluation of four methods of flexor tendon repair for postoperative active mobilization. Plast Reconstr Surg 2001;107:742-9.

14. Kessler I. The "grasping" technique for tendon repair. Hand 1973;5:253-5.

15. Urbaniak JR, Cahill JD, Mortenson RA. Tendon suturing methods: analysis of tensile strength. American Academy of Orthopaedic Surgeons Symposium on Tendon Surgery in the hand. St. Louis: CV Mosby; 1975. p. 70-80.

16. Tsuge K, Yoshikazu I, Matsuishi Y. Repair of flexor tendons by intratendinous tendon suture. J Hand Surg Am 1977;2: 436-40.

17. Barmakian JT, Lin H, Green SM, et al. Comparison of a suture technique with the modified Kessler method: resistance to gap formation. J Hand Surg Am 1994;19:777-81.

18. Cao Y, Tang JB. Biomechanical evaluation of a four-strand modification of the Tang method of tendon repair. J Hand Surg Br 2005;30:374-8.

19. Zidel P. Tendon healing and flexor tendon surgery. In: Thorne CH, editor. Grabb and Smith's plastic surgery. Philadelphia: LWW; 2007. p. 803-10.

20. Silfverskiold KL, May EJ. Flexor tendon repair in zone II with a new suture technique and an early mobilization program combining passive and active flexion. J Hand Surg Am 1994; 19:53-60.

21. Wang B, Xie RG, Tang JB. Biomechanical analysis of a modification of Tang method of tendon repair. J Hand Surg $\mathrm{Br}$ 2003;28:347-50.

22. Tang JB, Zhang Y, Cao Y, et al. Core suture purchase affects strength of tendon repairs. J Hand Surg Am 2005;30:12626.

23. Chang P. Repair and grafting of tendon. In: Mathes SJ, editor. Plastic surgery. Philadelphia: Saunders Elsevier; 2006. p. 591-603.

24. Becker H, Orak F, Duponselle E. Early active motion following a beveled technique of flexor tendon repair: report on fifty cases. J Hand Surg Am 1979;4:454-60.

25. Hashimoto T, Thoreson AR, An KN, et al. Comparison of step-cut and Pulvertaft attachment for flexor tendon graft: a biomechanics evaluation in an in vitro canine model. J Hand Surg Eur Vol 2012;37:848-54. 